\title{
Amino Acid Transport Systems of Lysosomes: Possible Substitute Utility of a Surviving Transport System for One Congenitally Defective or Absent
}

\author{
Halvor N. Christensen
}

Received February 2, 1988

\begin{abstract}
Ways in which other transport systems may compensate for one that is genetically defective are considered. Comparisons of the transport systems of organelles (here the lysosome) with the transport system at the plasma membrane has significant implications for chemotherapy.
\end{abstract}

KEY WORDS: diffusive passage; cystinosis; cystinuria; lysine; cystine; aspartate; glutamate; cysteamine; thiocholine; tyrosine; transport systems; Hartnup's disorder; amino acid transport; lysosomes.

Some views have been held for many years that various cellular organelles, and even to some extent the cell itself, are surrounded by membranes somewhat porous to various hydrophilic metabolites. These molecules were supposed largely to diffuse through holes or non-specific aqueous channels, with only occasional and often inconsistent concern about what dimensions such pores must have to explain the supposedly free passage of a variety of hydrophilic molecules. Although not to be excluded entirely, such diffusive passage was held to occur, in the case of the plasma membrane, to degrees that at times seemed to me unproved, excessive and inconsistent, in superimposition to the more or less specific mediated transport otherwise under measurement. I had questioned a parallel explanation that held the cystine molecule slightly too large for diffusive passage to avoid its accumulation in the lysosomes of various cells in cystinosis. The opportunity to challenge this historic view of the mode of escape of amino acids from the lysosome came through a collaboration with Dr Jess G. Thoene, but only after decisive evidence had already been obtained by Gahl et al. (1) that the genetric defect in nephropathic cystinosis lies in the absence of a competent membrane transport carrier comparatively specific to cystine in the leucocyte lysosome. Through the competent hands of Dr Ronald Pisoni (2) failure of this

Department of Biological Chemistry, The University of Michigan Medical School, Ann Arbor, Michigan 48109, USA. 
transport system was shown in my laboratory also to be accountable for cystine accumulation, in the same disease, in human skin fibroblasts.

My subsequent interest has proceeded principally along the lines of the question whether the lysosomal membrane has an extensive set of such transport systems, in analogy to the set shown for the plasma membrane (3). Other organelles, including for example the hepatic mitochondrion and epithelial brush borders, have shown apparently different sets of such systems. We proceeded to characterize a system by which basic amino acids can be exchanged, one molecule for another, possibly a different but analogous one, across the fibroblast lysosomal membrane. This technique of looking for specific mediated exchange between two transport analogues across a biological membrane is widely but not universally available in the transport field. The pursuit of this problem has also been facilitated by the development of a method for selectively loading lysosomes with an amino acid supplied as its methyl ester, a procedure adapted to this purpose by Reeves (4). On the entry of this comparatively lipid-soluble derivative into the organelles, the esterase activity of the lysosome releases the free amino acid from its ester, leading to the trapping of the amino acid rather specifically in the lysosome, even in the presence of other contaminating organelles. In the present case the exodus of ${ }^{14} \mathrm{C}$-labeled lysine was shown to be accelerated by the external presence of unlabeled lysine through the transmembrane exchange of the two species. Similar experiments were made for exchanges between lysine and arginine, with one of these labeled, to further characterize the new transport system. This transport system for cationic amino acids proved equally functional in the normal and cystinotic lysosome (2).

Gahl and other collaborating investigators have summarized recently (5) a broad study of the therapeutic value of administrating of cysteamine in cystinosis. The action of this monodecarboxylation product of cysteine was correctly attributed to its entry into a sulfhydryl-disulfide exchange reaction with the accumulated lysosomal cystine, a reaction which initially was incorrectly held to generate amino acid molecules small enough to escape via the hypothetical pores of that organelle. Our laboratory (2), again in the hands of Pisoni, proceeded to show that the critical therapeutic removal of cystine actually occurs instead in a modified form by the new transport system for basic amino acids. The mixed disulfide generated by the sulfhydryl-disulfide exchange, being itself a cationic amino acid, in effect a monodecarboxylation product this time of cystine, proved a substrate for the newly characterized system for transporting such amino acids Exodus of ${ }^{14} \mathrm{C}$-lysine could be shown to be stimulated by the external presence of the mixed disulfide of cysteine and cysteamine. Thus the therapeutic utility of cysteamine acquired a new explanation through its generation of a specifically transportable derivative of cystine.

Our initial supposition was that the new transport system would be much like the one present in the plasma membrane of various cells, and also in the brush borders of epithelial tissues. This system we had designated $\mathrm{y}^{+}$, the plus sign serving to indicate its requirement for a net charge of plus one on the amino acid molecules ordinarily accepted by it. Some years later $(6,7)$, however, we discovered, along with that feature, an unexpected further characteristic of this system in virtually every tissue tested that an accompanying sodium ion could 
serve as a surrogate for the plus one charge on the sidechain of cationic amino acids. Hence certain dipolar ("neutral") amino acids could also be transported by system $\mathrm{y}^{+}$, leaving the plus sign perhaps somewhat ambiguous.

At this point we were again surprised to discover that the new lysosomal system for basic amino acids showed no detectable corresponding reactivity with dipolar amino acids (8). Alpha- $\mathrm{N}$-methyl arginine strongly inhibits $\left[{ }^{14} \mathrm{C}\right]$ arginine uptake by lysosomal system $\mathrm{c}$ in contrast to its lack of effect on system $\mathrm{y}^{+}$in the plasma membrane. Furthermore, the distal $\mathrm{N}$ atom of lysine could be rendered quaternary (hence permanently cationic) by methylation, a change not acceptable to system $\mathrm{y}^{+}$. Gradually it became clear to us that the lysosomal system is quite unlike that of the plasma membrane (8). Indeed, if we selected an analog to cysteamine with quaternary $\mathrm{N}$ (namely thiocholine), we could produce with lysosomal cystine a reaction entirely analogous to that obtained with cysteamine, but with the possible advantage that the new mixed disulfide can pass the lysosomal membrane via the cationic amino acid system, without at the same time serving as an inhibitor for the approximately corresponding transport system of the plasma membrane of the fibroblast.

Thus our team has brought forth the concept of a novel therapeutic approach, setting up the service of a surviving transport system to carry out the function of one that is congenitally absent or defective (8). To explore this strategy fully, one needs to look also for any alternative route for the membrane transport, in this case of cystine per se, that survives in the congenital defect, which might somehow be caused to serve effectively enough to compensate for the defect. For example, exposure to $43^{\circ}$ accelerates the subsequent loss of cystine, even from cystinotic fibroblast lysosomes, perhaps because an as yet unknown alternative system is intensified (Lemons et al., 8a). I have emphasized elsewhere (8b) the technology of kinetic challenges to the supposed homogeneity of any pertinent transport system, essential to the full description of the movement of a metabolite.

Before extension to other transport defects, of the strategy of the use of a surviving transport system to substitute for a missing one, let me examine further a pertinent question: To what degree might various lysosomal transport systems prove different from those in the plasma membrane in ways that could prove biologically or therapeutically advantageous?

So far our experiments had, as mentioned, been assisted by access to the measure of transport stimulation by the transmembrane exchange of transport analogs. This phenomenon had made it unnecessary to purify the lysosomes to a high degree. Now we needed to turn to transport systems that seemed not to show this phenomenon. To fill the resulting gap, Pisoni adapted the known technology of purifying lysosomes by Percoll gradient sedimentation. This technology allowed direct measure of the fluxes of various amino acids into or out of the organelle, although with more variance than one would wish. Incidentally, experience has taught that either of these opposed fluxes can prove useful for characterizing the transport facilities of a biomembrane. Three transport systems, $e, f$, and $p$, involved in the movements of such small dipolar amino acids as proline, alanine and the like were thus characterized by Pisoni et al. (9), as illustrated in Fig. 1. Subsequently, Collarini et al. characterized the mode of 


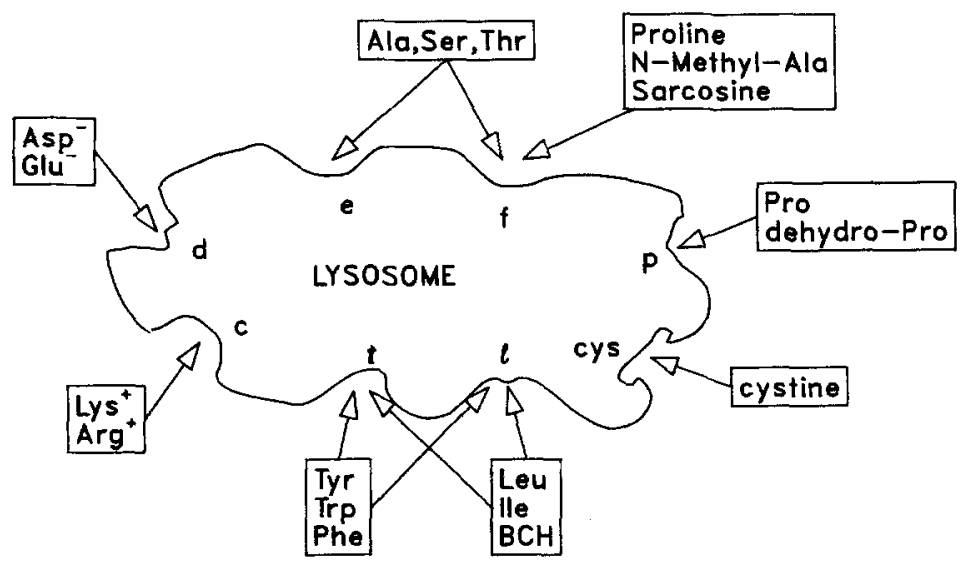

Fig. 1. Provisional representation of the amino acid transport systems detected so far for human fibroblast lysosomes. In the FRTL cell line (25) the designation $h$ may apply either to the component here marked $t$ or to the total action of $t+h$. Sketch drawn by R. L. Pisoni as an extension of Fig. 9 in ref. 9 .

mediation of the transport of asparate and glutamate (10), which proved attributable to a single $\mathrm{Na}^{+}$-dependent system resembling system $\mathrm{x}_{\mathrm{AG}}^{-}$of the plasma membrane $(11,12)$, although with further study clear differences again emerged. Several other systems participating along with this one in plasma membranes of various cells were not detected, however, for the lysosome.

Our attention turned then, in the hands of Stewart et al. (13), to the mode of movement of the dipolar amino acids with bulky sidechains, for which the facilities in plasma membranes, namely systems $\mathrm{L}$ and $\mathrm{T}$, are largely sodium-ionindependent and quite distinct. In the meantime, Bernar et al. (14) had partially characterized, under the designation lysosomal system $h$, the transport of L-tyrosine into a cultured thyroid cell line designated FRTL-5. A number of amino acids with bulky sidechains, including leucine and tryptophan, inhibited tyrosine uptake. Stewart soon obtained evidence that the competitive transport interactions with other bulky dipolar amino acids corresponded for the fibroblast lysosome to the participation of not one but at least two different transport systems, even though the total uptake both of phenylalanine and leucine correspond to a single rectangular hyperbola (13). At this point the situation seems to us reminiscent of that seen in human red blood cells, where system $L$ serves for a large group of such amino acids, but a different system, $T$, prefers within this group the aromatic amino acids, tyrosine, phenylalanine, and tryptophan (see review, ref. 3). We seek model substrates specific to each of these systems to improve the evidence for a parallel situation in the lysosome.

Although we began with a tendency to apply to the lysosomal systems the designations suggested by analogies of various strengths with the systems for plasma membranes, for example lysosomal systems $\mathrm{y}^{+}$, A, asc, etc., we soon came to see that these choices might bias our search for differences, as we tended to emphasize such manifestly imperfect analogies. The biological probability of their separate genetic determination, and also of their separate regulation, has led 
Table 1. Search for parallelisms between somewhat analogous pairs of transport systems of plasma and lysosomal membranes

\begin{tabular}{|c|c|c|c|c|}
\hline $\begin{array}{l}\text { Amino acids } \\
\text { transported }\end{array}$ & $\begin{array}{l}\text { Plasma membran } \\
\text { Designation }\end{array}$ & $\begin{array}{l}\text { e system } \\
\left(\mathrm{Na}^{+} \text {-dep? }\right)\end{array}$ & $\begin{array}{c}\text { Compared lysosomal } \\
\text { system }\end{array}$ & $\begin{array}{l}\text { Reasonably } \\
\text { analogous? }\end{array}$ \\
\hline Smaller dipolar & No analog detected & - & Lysosomal system $\mathrm{f}^{9}$ & \\
\hline Proline, not ala & Iminoglycine? & Yes & Lysosomal system $p^{9}$ & Dubious \\
\hline $\begin{array}{l}\text { Small dipolar \& } \\
\text { small anionic }\end{array}$ & ASC & Yes & Not detected & \\
\hline $\begin{array}{l}\text { Wide range of } \\
\text { dipolar \& cationic }\end{array}$ & $\mathbf{B}^{0,+}$ & Yes & Not detected & \\
\hline $\begin{array}{l}\text { Wide range of } \\
\text { dipolar \& cationic }\end{array}$ & $b^{0,+}$ & No & Not detected & \\
\hline Bulky dipolar & $\mathrm{L}$ & No & Lysosomal system $\mathrm{I}^{12,14}$ & Yes \\
\hline Aromatic & $\mathrm{T}$ & No & $\begin{array}{l}\text { Lysosomal system } \mathrm{t}^{12} \\
\text { or } \mathrm{h}^{25}\end{array}$ & Yes \\
\hline Smaller dipolar & asc & No & Lysosomal system $\mathrm{e}^{9}$ & Possibly \\
\hline Cystine, tetrapolar & (E. coli) & No & Lysosomal cystine & \\
\hline Cystine, glutamate & $\mathrm{x}_{\mathrm{C}}^{-}$ & No & Not detected & \\
\hline Glutamate, aspartate & $\mathrm{x}_{\mathrm{AG}}^{-}$ & Yes & Lysosomal system $\mathrm{d}^{8}$ & Yes \\
\hline $\begin{array}{l}\text { Cationic (dipolar } \\
+\mathrm{Na} \text { ) }\end{array}$ & $\mathrm{y}^{+}$ & No & Not detected & \\
\hline $\begin{array}{l}\text { Catonic (not } \\
\text { dipolar) }\end{array}$ & Blastocyst $^{21}$ & No & Lysosomal system $c^{8}$ & Yes \\
\hline
\end{tabular}

us now to propose provisional designations that may instead go temporarily too far in ignoring the parallelisms between systems in the two types of membranes. As we look over our results at this stage (Table 1, above) we believe four or five of the parallelisms viewed post hoc are indeed considerable, although in no case do we conclude for identity of any pair of systems in the two membranes. Reconsideration of one or more provisional designations may come to be counseled by more striking parallelisms. Until the functional transport proteins have been sequenced, presumably with recognition of the complementary sequences in DNA, are we likely to understand, through the recognition of homologies of structure, the differences and the parallelisms. I suspect that the general similarities and yet the differences in membrane transport functions among tissues and organelles at various degrees of differentiation will prove very interesting and important.

The existence of these several lysosomal transport systems suggest that sulfhydryl compounds other than aminothiols, e.g. mercaptoalkanes, mercaptoalkyl-sulfonates, or mercaptoalkyl carboxylic acids might be tested for their ability to stimulate exodus of cystine from lysosomes. Let me return then to consider clues as to other ways in which a surviving membrane transport system might be put into service in place of one congenitally deficient. Perhaps an earlier observation may suggest that supplementations between the service of epithelial transport systems assists our nutriture more often than we may realize. In Hartnup's disorder the defect of a broad-range system serving in the epithelial absorption of various dipolar amino acids leads to urinary losses and retarded intestinal absorption of several such amino acids. The disorder can deserve the name disease only when the moderate wastage of tryptophan it produces leads to 
a pellegra-like dermatitis. Tests in a single subject with this disorder by phenylalanine and tryptophan feeding showed abnormally small responses in the rise of the circulating levels of these amino acids. If, however, glycyltryptophan or phenylalaninylphenylalanine were fed instead, much larger rises were produced in the plasma levels of free tryptophan and phenylalanine (15). In these tests one of the known intestinal transport systems for dipeptides or oligopeptides was logically taken to serve in place of the defective system for free amino acids in Hartnup's disorder. Peptidase action in the epithelial cells usually, but not always, follows peptide absorption into these cells. Such nutritive substitutions of transport facilities might come into play spontaneously more often than we may realize, perhaps in some cases compensating for inborn transport defects that would otherwise be less benign than Hartnup's disorder (see 16).

Recent evidence may suggest ways to enhance the service of a surviving transport system in compensation for that deficient in cystinuria, a disease of course dramatically unlike cystinosis. In the transport interactions defective in this disease, cystine is included among the diamino monocarboxylic acids; i.e., one of its carboxyl groups is apparently treated by the affected renal transport system as though it were received in the uncharged state (3). As I have already described for system $\mathrm{y}^{+}$, the two brush-border transport systems in question, namely the one congenitally absent in cystinuria and the one apparently serving in a varying degree of compensation, appear to be reactive also in the presence of sufficient levels of sodium ion with certain dipolar amino acids. This reactivity has also been described for the MDCK renal epithelial cell line (17). This effect had first been seen as a largely homoserine-dependent inhibition of homoarginine transport by $\mathrm{Na}^{+}$, and by a $\mathrm{Na}^{+}$- or $\mathrm{Li}^{+}$-dependent inhibition of homoarginine uptake by homoserine or its 5-carbon homolog (7). The effect was subsequently extended to glutamine in experiments on human skin fibroblasts (18), although the shorter analog, serine, was always found relatively inactive. The interaction is also manifested by a trans-stimulated exchange across the plasma membrane of the monoamino acid plus sodium ion, on the one hand, for the diamino acid alone, on the other. Sodium ion inhibition did not, furthermore, fall to zero as the homoserine level was reduced to zero; i.e., sodium ion by itself inhibits arginine uptake (7). To secure the effect of the dipolar amino acids one needs a hydroxyl group or its terminal carbon 4 or 5 , or a carboxamide group on carbon 4. This spatial requirement points to a bonding to the sodium ion by otherwise unshared electrons of those two sidechain structures when they are at a suitable distance from the alpha carbon atom.

An enhancement of the renal resorption of cystine in human cystinuria by the feeding of glutamine has been investigated as a way to decrease the risk of renal stone formation, a recurrent problem in such patients. That effect is obtained, however, only when the sodium excretion is somewhat elevated (19). Indeed, sodium restriction alone may serve quite well by itself to reduce stone formation. I suggest, however, that administration of a sèlected amino acid may yet come to represent a helpful therapeutic aid in cystinuria, even when efforts have been made to decrease $\mathrm{Na}^{+}$intake, a possibility that should probably not be dismissed until the 4- and 5-carbon homologs of serine have been tried at readily 
feasible decreases in sodium-ion excretion rates. Homoserine has been administered to human subjects at high dosages, sufficient indeed to produce evidence of hemodilution, apparently without other significant unfavorable effects (20). The hypothesis lying provisionally behind this suggestion is that such selected amino acids plus sodium ion may exchange for renal tubular cystine via a surviving brush-border transport system of the $\mathrm{y}^{+}$type, to provide a significantly enhanced prophylaxis against stone in cystinuria. In the meantime, a watch should probably be kept for any more promising opportunities to apply the title strategy.

The comparison obtained so far for lysosomal and plasma membrane systems is now concluded with reference to Table 1 . The differences seen are somewhat as follows: $\mathrm{Na}^{+}$-dependence has not been seen with any of the lysosomal systems, but effects of an outward-directed $\mathrm{Na}^{+}$-gradient have not yet been excluded. In that connection, system L uptake by fibroblasts seems to be enhanced by the intracellular presence of $\mathrm{Na}^{+}$along with an exchangeable amino acid (22). Lysosomal system $\mathrm{f}$ tolerates $\mathrm{N}$-methylation of the amino group, whereas system e does not (9), a pattern reminiscent of the relation between systems $A$ and either ASC or asc. Although variants of system A, as seen in transformed hepatocyte lines $(23,24)$, may ultimately need to be considered, parallelisms between lysosomal system $\mathrm{f}$ and plasma membrane systems for smaller dipolar amino acids are not yet impressive. For example, 2-aminoisobutyrate has little effect on proline uptake by lysosomal system f. Although lysosomal system e serves as the main route of passage of alanine, serine, and threonine, which three show mutually competitive uptake, that passage is scarcely affected by the presence of proline, or decreased by the lowering of the $\mathrm{pH}$ or replacement of $\mathrm{Na}^{+}$, as seen for system ASC. Comparison of system e with $\mathrm{Na}^{+}$-independent system asc is more plausible than with ASC, but remains incomplete.

The duality in the mediation of the membrane transport of bulky dipolar amino acids, by systems $T$ and $L$ in red blood cells, for example, and by systems $t$ and 1 in the lysosomal membrane (13), as tested by the inhibition of a wide range of bulky aromatic and non-aromatic amino acids (cf. 14), may be emerging as an interesting parallelism, although model analogs are needed to enhance the precision of that discrimination in the lysosome. D-Tryptophan is the most effective inhibitor of the system by which L-tryptophan is preferentially taken up, while norleucine or $\mathrm{BCH}$ fully inhibits the saturable component of L-leucine lysosomal transport. If indeed two systems are involved here in the FRTL cell as well as in the fibroblast, the provisional designations $t$ and 1 may prove more suitable than $\mathrm{h}(25)$.

Cystine transport by the lysosomal membrane appears to call for recognition of all four of its potentially charged groups, a requirement not yet seen in higher animal plasma membranes, although cystine transport into $E$. coli appears to show that feature (26). Otherwise cystine seems to be received as one or the other of the two plausible tripolar ions (3), e.g. as a glutamate analog by system $\mathrm{x}_{\mathrm{C}}^{-}$.

The system transporting aspartate and glutamate across the lysosomal membrane is similar to the ubiquitous system $X_{A_{G}}^{-}(11)$, but differs clearly in these respects: (a) the stereoselectivity for the $L$ isomers is weaker than in plasma 
membranes, but as strong for one as for the other dicarboxylic amino acid; (b) 3-aminoglutaric acid is a much weaker inhibitor of the lysosomal than for the plasma membrane system; (c) the $\mathrm{N}$-methyl and omega-hydroxamate derivatives of aspartate and glutamate are inhibitory, the aspartate derivatives being the more effective, whereas in the plasma membrane only the aspartate derivatives are effective. In agreement with the indication of a longer space available for an anionic sidechain in the lysosome given by the hydroxamates, alpha-aminoadipic acid is a stronger inhibitor of the lysosomal than of the plasma membrane system; (d) as seen also for lysine in system c, methylation of the alpha amino group of aspartate or glutamate has little effect on their transport reactivity in both cases in the plasma membranes of various tissues. As recorded in Table 1 , we no longer regard system $\mathrm{c}$ as a good analog of system $\mathrm{y}^{+}$, although substitution of a single amino acid residue in the transporter molecules might conceivably prove to account for the considerable difference in cationic amino acid transport observed between the two membranes. It seems rather likely to me that other tissues, e.g. the intestinal brush border or the preimplantation mouse blastocyst (21) may contain a closer analog of lysosomal system $c$ with respect to its rejection of dipolar amino acids.

In conclusion I urge that learning more about the specific transport functions of various cellular membranes may allow recognition of further congenital defects in these functions, and allow further opportunities for therapeutic use of an unimpaired transport system in substitution for the congenitally defective one.

\section{ACKNOWLEDGEMENTS}

Support acknowledged from Grants DK32281 and HD01233, National Institutes of Health.

\section{REFERENCES}

1. Gahl, W. A., Tietze, F., Bashan, N., Steinherz, R. and Schulman, J. D. (1982). J. Biol. Chem. 257:9570-9575.

2. Pisoni, R. L., Thoene, J. G. and Christensen, H. N. (1985). J. Biol. Chem. 260:4791-4798.

3. Christensen, H. N. (1984). Biochim. Biophys. Acta 779: 255-269.

4. Reeves, J. P. (1979). J. Biol. Chem. 254:8914-8921.

5. Gahl, W. A., Reed, G. F., Thoene, J. G., Schulman, J. D., Rizzo, W. B., Jonas, A. J., Denman, D. W., Schlesselman, J. J., Corden, B. J. and Schneider, J. A. (1987). N. Engl. J. Med. 316:971-977.

6. Christensen, H. N. and Antonioli, J. A. (1969). J. Biol. Chem. 244:1497-1504

7. Christensen, H. N., Handlogten, M. E. and Thomas, E. L. (1969). Proc. Nat. Acad. Sci. U.S. 63: $948-955$.

8. Pisoni, R. L., Thoene, J. G., Lemons, R. M. and Christensen, H. N. (1987a). J. Biol. Chem. 262: $15011-15018$.

8a. Lemons, R., Pisoni, R. L., Christensen, H. N., and Thoene, J. G. (1986). Biochim. Biophys. Acta, 884:429-434.

8b. Christensen, H. N. (1989). Methods in Enzymol., in press.

9. Pisoni, R. L., Flickinger, K. S., Thoene, J. G. and Christensen, H. N. (1987b). J. Biol. Chem. 262:6010-6017. 
10. Collarini, E. J., Pisoni, R. L., and Christensen, H. N. (1988). FASEB J. (in press).

11. Makowske, M. and Christensen, H. N. (1982). J. Biol. Chem. 257: 5663-5670.

12. Christensen, H. N. and Makowske, M. (1983). Life Sci. 33:2255-2267.

13. Stewart, B. H., Collarini, E. J., Pisoni, R. L., and Christensen, H. N. (1988). FASEB J. (in press).

14. Bernar, J., Tietze, F., Kohn, L., Bernardini, I., Harper, G. S., Grollman, E. F., and Gahl, W. A. (1986). J. Biol. Chem. 261:17107-17112.

15. Asatoor, A. M., Cheng, B., Edwards, K. D. G., Lant, A. F., Mattews, D. M., Milne, M. D., Navab, F. and Richards, A. J. (1970). Gut 11:380-387.

16. Adibi, S. A., Fekl, W., Furst, P. and Oemke, M. (1987). In Beitrage zur Infusions Therapie und Klinische Ehnahrüng, Vol. 17, S. Karger, Basel.

17. Sepúlveda, F. V. and Pearson, J. D. (1985). J. Cell. Physiol. 123:144-150.

18. White, M. F., Gazzola, G. C. and Christensen, H. N. (1982). J. Biol. Chem. 257:4443-4449.

19. Jaegger, P., Portmann, L., Saunders, A., Rosenberg, L. E. and Thier, S. O. (1987). N. Engl. J. Med. 314:1120-1123.

20. Gaul, G. E., Wada, Y., Schneiderman, K., Rassin, D. K., Tallan, H. H. and Sturman, J. A. (1971). Pediatr. Res. 5:265-273.

21. Van Winkle, L. J., Campione, A. L. and Gorman, J. M. (1988). J. Biol. Chem. 263 (in press). Cited with permission of the authors.

22. Gandolfi, S. A., Petronini, R. G. and Borghetti, A. F. (1985). Trans. Biochem. Soc. 13:701-702.

23. Handlogten, M. E., Garcia-Cañero, R., Lancaster, K. T. and Christensen, H. N. (1981). J. Biol. Chem. 256: 7905-7909.

24. Dudeck, K. L., Dudenhausen, E. E., Chiles, T. C., Fafournoux, P. and Kilberg, M. S. (1987). J. Biol. Chem. 262: 12565-12569.

25. Harper, G. S., Kohn, L. D., Bernardini, I., Bernar, J., Tietze, F. and Gahl, W. A. (1988). J. Biol. Chem. 263 (in press).

26. Lieve, L. and Davis, B. D. (1965). J. Biol. Chem. 240:4362-4369. 